Delphine Pasques

\title{
Zum Gebrauch von ein in den althochdeutschen Nominalgruppen: Grammatikalisierung oder Systematisierung?
}

Im Neuhochdeutschen lässt sich nach Jean Fourquet (1992) (vgl. Briu 2001) eine Nominalgruppe durch die beiden semantischen Kategorien der Definitheit (Opposition definit nicht definit) und des Numerus definieren. Unter Numerus versteht Fourquet über die Opposition Singular Plural hinaus die Opposition zwischen dem „Zählbaren“ und dem „Nicht-Zählbaren“, welche weder lexikalisch (im Sprachsystem) noch außerlinguistisch (in der „Welt“) definitiv festgelegt ist, sondern auch von der Perspektive des Sprechers abhängt (vgl. auch Valentin 1984; Pasques 2015). ${ }^{1}$ Daraus ergibt sich folgende Struktur für die Nominalkategorie Numerus im Neuhochdeutschen (siehe Tab. 1).

Dem Morphem ${ }^{2}$ ein kommt in diesem System die Funktion zu, die Opposition zwischen dem Singular 1 (oder SG1, d.h. dem Singular des Nicht-Diskreten, des Kontinuierlichen: Ø Brot, Ø Freiheit) und dem Singular 2 (oder SG2, d.h. dem Singular des Diskreten, des Diskontinuierlichen: ein Brot, eine Freiheit) zu markieren. $\mathrm{Zu}$ bemerken ist, dass ein in Fourquets Modell keine Funktion innerhalb der anderen Nominalkategorie, der Definitheit, zukommt. Nichtdefinitheit (und nicht „Indefinitheit“) liegt vor, sobald keine Markierung der Definitheit verwendet wird (das Brot Ø Brot, ein Brot). ${ }^{3}$

1 Vgl. auch Georges Kleiber (1989: 4), der in dem Sinne von „conditionnement référentiel“ spricht, d.h. von der Art und Weise, wie der Referent „,verpackt“ wird.

2 Unter „Morphem“ verstehe ich in Anlehnung an Martinet und u.a. Soutet eine Phonemfolge, die nicht in kleinere Einheiten zerlegbar ist und eine feste Bedeutung besitzt (Martinet verwendet aber den Ausdruck „monème“, der heute kaum benutzt wird, vgl. Martinet 1970: 15 und Soutet 1995: 132).

3 Eigentlich sollte das Nullzeichen für den nicht definiten SG1 zweimal vorkommen: In dem SG1 $\emptyset \emptyset$ Brot verweist das erste Nullzeichen auf die Abwesenheit von das bzw. auf die Nichtdefinitheit, das zweite auf die Abwesenheit von ein bzw. auf die Nichtdiskretheit. Für den SG2 sollte das Nullzeichen einmal vorliegen: In Ø ein Brot verweist es auf die Abwesenheit von das bzw. auf die Nichtdefinitheit, während ein die Diskretheit markiert. 
Tab. 1: Nominalkategorie Numerus im Neuhochdeutschen

\begin{tabular}{lll}
\hline $\begin{array}{l}\text { Nicht-zählbar } \\
\text { (nicht diskret) }^{4}\end{array}$ & $\begin{array}{l}\text { Zählbar } \\
\text { (diskret) }\end{array}$ \\
\hline Singular 1 (SG1) & Singular 2 (SG2) & Plural \\
\hline$\varnothing$ Brot & ein Brot & zwei Brote, $\varnothing$ Brote \\
$\varnothing$ Freiheit & eine Freiheit & zwei Freiheiten, $\varnothing$ Freiheiten $^{6}$ \\
\hline
\end{tabular}

Im Althochdeutschen ist die Opposition zwischen SG1 und SG2 noch nicht systematisch markiert. Im Isidortraktat (790) kann die minimalste Form einer NG (Ø $N)$ sowohl für den SG1 als auch für den SG2 stehen. ${ }^{7}$ In Otfrids Evangelienbuch (860) lassen sich im Vergleich zum Isidortraktat und zur Tatianbilingue (830) quantitative und qualitative Entwicklungen im Gebrauch von ein als Marker der Opposition SG1 SG2 beobachten (Kapitel 1). Inwieweit tritt dabei eine neue Funktion von ein auf? Ist die Markierung der Opposition SG1 SG2 durch ein schon Zwang

4 Ich ziehe den Terminus 'diskret' vor, weil 'zählbar' meiner Meinung nach voraussetzt, dass der Referent, auf den verwiesen wird, an sich zählbar oder nicht zählbar ist, während der Sprecher über die Möglichkeit verfügt, zwischen beiden Perspektiven zu wählen (ein Brot Ø Brot). 'Diskret' verstehe ich im mathematischen Sinne als 'abgesondert', aus lat. discernere 'unterscheiden, absondern, trennen’. Stark (2006: 64 und 296) spricht ihrerseits von „konturiert“ versus „diffus“.

5 Z.B. in: „Unser armer Philosoph zum Beispiel glaubt fest daran, dass der Mensch eine Freiheit besitzt“ (Eine Hommage an die Freiheit: Ein Drama in vier Generationen, von Beate Seifert, 2013, Books on Demand, 6); oder in einem Artikeltitel: Tätowierungen - eine Freiheit des Gläubigen? (www.folgemirnach.de/2015-04-taetowierungen-eine-freiheit-des-glaeubigen-a74.html, Stand: 29.6.2017).

6 Verschiedene Bezeichnungseffekte treten durch Pluralsetzung auf. Vgl. Beispiele aus dem Duden Online-Wörterbuch: besondere Freiheiten genießen; sich gewisse Freiheiten erlauben. (www. duden.de/rechtschreibung/Freiheit\#Bedeutung1, Stand: 29.6.2017). Vgl. das Buchtitel von Astrid Seele: Römische Übersetzer. Nöte, Freiheiten, Absichten. Verfahren des literarischen Übersetzens in der griechisch-römischen Antike (Darmstadt, 1995). Vgl. in dem wissenschaftlichen Kontext der Thermodynamik: „Die Gibbssche Phasenregel ergibt daher zwei Freiheiten“ [d.h. Druck und Temperatur], aus Thormann (2013: 2).

7 Beispiel für den durch $\emptyset N$ markierten SG1 im Isidortraktat:

$>$ Oxsso auh endi leo dhar ezssant samant spriu< (Isidortraktat IX,9) „Der Ochse und auch der Löwe essen dort Spreu zusammen“.

Beispiel für den durch Ø $N$ markierten SG2 im Isidortraktat:

$>$ Chindh uuirdit uns chiboran, sunu uuirdit uns chigheban< (Isidortraktat V,1) „Ein Kind ist uns geboren, ein Sohn ist uns gegeben worden“. 
geworden? Mit anderen Worten, geht es gegebenfalls um eine Systematisierung oder um eine Grammatikalisierung von ein? (Kapitel 2). ${ }^{8}$

\section{1 ein als Marker der Opposition SG1 SG2 bei Otfrid: die Fakten}

Sehr aufschlussreich für diese Korpusuntersuchung ist der Vergleich von Otfrids Evangelienbuch (860) mit der Tatianbilingue (830). Beide Werke behandeln im großen und ganzen dieselbe Materie (das Leben Jesu), so dass bestimmte Textstellen aus den Evangelien sich besonders leicht vergleichen lassen. Wichtig ist dabei mitzuberücksichtigen, dass die Tatianbilingue eine Übersetzung aus dem Latein darstellt, während Otfrid die Harmonie selber gedichtet hat, wahrscheinlich direkt im Althochdeutschen, so dass dieser Text weniger lateinabhängig ist.

Die zwei häufigsten Vergleichskonstellationen zwischen beiden Korpora sind folgende:

1. Eine nicht definite NG taucht in der Tatianbilingue mit der Form $\emptyset N$ (s.u. Øman blintan fon giburtj „[einen] blindgeborenen Mann“), im entsprechenden Textzusammenhang bei Otfrid mit der Form ein $N$ auf (einan man blintan giboranan):

(1) furfarentj gisah Øman blintan fon giburtj (Tatian 220,13) gisah tho druhtin einan man blintan giboranan (Otfrid III 201 ) et praeteriens Jesus vidit hominem caecum a navitate (Johannesevangelium 9,1) „Und Jesus ging vorüber und sah einen Menschen, der blind geboren war“

In beiden Korpora zielt die fettgedruckte NG auf ein spezifisches, zum ersten Mal erwähntes Diskursobjekt, und zwar den Blinden, dem Jesus begegnet. Dieser SG2 (diskrete Einheit), dem im Kontext eine spezifische Interpretation zukommt, wird bei Otfrid durch ein explizit markiert, während im Tatian eine solche Signalisierung ausbleibt. Bei Otfrid weisen solche Ersterwähnungen eines Diskursobjektes, die anschließend mehrmals wiederaufgenommen werden, immer die Form ein $N$

8 Für den Unterschied zwischen Grammatikalisierung und Systematisierung vgl. den Anfang von Kapitel 2.3. 
auf; in der Tatianbilingue ist eine solche Tendenz nicht $\mathrm{zu}$ verzeichnen, obwohl ein in nicht definiten $\mathrm{NG}^{9}$ auch schon belegt ist.

2. Eine nicht definite NG taucht sowohl im Tatian als auch bei Otfrid mit der Form $\emptyset N$ auf. Das Morphem ein fällt in beiden Korpora aus, obwohl das entsprechende Diskursobjekt auch als SG2 (diskrete Einheit) zu verstehen ist:

(2) Ih quidu iu thaz iogiuuelih thiethar gisihit Øuuib sie zigeronne iu habet sia forlegana in sinemo herzen (Tatian 63,20)

Ih, quad, avur sagen iu, ther Øwib biscówot zi thíu thaz sar in thémo frist zi thíu nan es gilúste... (Otfrid II 193)

Ego autem dico vobis, quia omnis qui viderit mulierem ad concupiscendum eam, jam moechatus este am in corde suo (Matthäusevangelium 5, 28-29) „Ich aber sage euch: Wer eine Frau auch nur lüstern ansieht, hat in seinem Herzen schon Ehebruch mit ihr begangen“

Das in diesem Kontext bezeichnete Diskursobjekt (SG2) ist nicht spezifisch zu interpretieren: Es geht nicht um eine bestimmte Frau, die lüstern angesehen wird, sondern um die Potenzialität eines solchen Prozesses bzw. um eine beliebige Frau, die von einem beliebigen Mann ${ }^{10}$ lüstern angesehen wird. Die finite Verbform im Relativsatz (siehe gisihit im Tatian, biscówot bei Otfrid) steht im Indikativ Präsens, hier als Präsens der Allgemeingültigkeit zu interpretieren. Es handelt sich um eine generelle Warnung, die nicht zeitlich-räumlich verankert ist. In einem solchen nicht aktualisierenden Kontext, ${ }^{11}$ in welchem den Diskursobjek-

9 Vgl. gieng zi imo ein centenari bat inan... (Tatian 83,9) ju quam ein sculdheizo bi notthurfti heizo (Otfrid III 3 5)

Lat. accessit ad eum Øcenturio rogans eum... „,[da] trat ein Hauptmann an ihn heran und bat ihn...“ (Matthäusevangelium 8,5)

10 Auf den entsprechenden beliebigen Mann wird im Tatian durch die Kombination von Indefinitpronomen und Relativpronomen iogiuuelih thiethar ,irgendwelcher der“ verwiesen, bei Otfrid durch das Relativpronomen ther.

11 Marion Presslich (2000) spricht in diesem Sinne von „nicht restriktiven Kontexten“, die sich „von den restriktiven dadurch [unterscheiden], dass sie sich auf Handlungssituationen beziehen, die zeitlich und räumlich nicht eindeutig auf eine Begebenheit beschränkt sind. Der Referenzrahmen der beteiligten Nomen bzw. Gegenstände ist somit beliebig groß, d.h. nicht begrenzt“ (ebd.: 51). Ich ziehe den Terminus „desaktualisierend“ bzw. „aktualiserend“ vor, der sich deutlicher auf die zeitlich-räumliche Verankerung der von den NG enkodierten Diskursobjekte bezieht. Die Gesamtheit der an einer Äußerung beteiligten Zeichen trägt dazu bei, die Diskursobjekte als aktuell oder nicht emergieren zu lassen, d.h. sowohl die Form der NG wie auch Tempus und Modus der finiten Verbform, die Satzsyntax, der Äußerungstypus usw. (vgl. Pasques 2015, wo die unterschiedlichsten Zeichenkombinationen, wie sie im Korpus belegt sind, hinsichtlich 
ten eine unspezifische Interpretation zukommt, taucht im Ahd. das Morphem ein nie auf.

In Otfrids Korpus bin ich auf eine einzige Okkurrenz von ein in einem desaktualisierenden Kontext gestoßen. Aufschlussreich ist, dass im entsprechenden Kontext das Morphem ein eher die Einzigkeit ausdrückt und akzentuiert wird:

(3) Soso éin man sih scal wérien joh héreron sinan nérien: so aht er io ginóto thero Kristes fianto! (Otfrid IV 17 13)

„Wie sich ein Mann wehren und seinen Herrn erretten soll, so verfolgte er die Feinde Christi ganz und gar“

In dem von soso eingeführten generalisierenden Vergleich, der in die aktuelle Handlung bzw. die Verteidigung Christi durch Petrus übergeht (vgl. die Korrelation soso ... so), wird die Einzigkeit (éin man/er) der Pluralität (thero Kristes fianto) gegenübergestellt. Die NG éin man taucht im generalisierenden Korrelat auf, sie verweist auf kein spezifisches Diskursobjekt, sondern auf jeden einzigen Kämpfer, der auch im ungerechten Kampf eingreifen soll (vgl. scal). Das Morphem ein kann also im Korpus Otfrids in einem desaktualisierenden Kontext verwendet werden, aber nur dann, wenn auf die Einzigkeit Nachdruck gelegt wird. ${ }^{12}$

Aus diesen beiden rasch skizzierten Vergleichskonstellationen ergibt sich Folgendes:

- Im Tatian dient das Morphem ein noch nicht der Markierung der Opposition SG1 SG2. Die Nominalsequenz $\emptyset N$ kann sowohl auf ein nicht diskretes Diskursobjekt (SG1) hinweisen, als auch auf ein diskretes Objekt (SG2); in einem aktualisierenden Kontext ist dieser SG2 als spezifisch (d.h. zeitlich-räumlich verankert: Ein bestimmter Referent ist gemeint) $\mathrm{zu}$ interpretieren; in einem desaktualisierenden Kontext hingegen als unspezifisch (d.h. noch nicht zeitlich-räumlich verankert: Ein beliebiger Referent, der später näher bestimmt wird oder nicht), oder als generisch (falls der Kontext eine generische Interpretation der NG auslöst). ${ }^{13}$

ihrer (des-)aktualisierenden Wirkung untersucht werden). Stark spricht ihrerseits von nichtspezifischen Kontexten, die sehr detailliert untersucht werden (Stark 2006: 122-125).

12 Das ist übrigens im Tatian schon der Fall, vgl.:

Quad in. ir niuuizzut îouuiht. noh nithenket bithiu uuanta uns bitherbisot thaz ein man ersterbe furi thaz folc. inti nalles al thiu thiota foruuerde (Tatian 135, 29) „Er sprach zu ihnen: ihr wisst noch bedenkt nicht, dass für uns besser ist, dass ein [einziger] Mann für uns stirbt, anstatt dass das ganze Volk verdirbt“.

$13 \mathrm{Zu}$ der Opposition spezifisch/unspezifisch vgl. Stark (2006: 42ff.). 
- $\quad$ Bei Otfrid erweist sich der Kontexttypus hinsichtlich der Markierung des SG2 durch ein als entscheidender Faktor. Wirkt der Kontext aktualisierend (z.B. durch die Zeit-Modus-Kombination Indikativ Präteritum), bzw. ist der SG2 spezifisch zu interpretieren, so nimmt eine nicht definite NG sehr regelmäßig die Form ein $N$ an. ${ }^{14}$ In einem desaktualisierenden Kontext hingegen tritt ein nur dann auf, wenn Nachdruck auf die Einzigkeit gelegt wird.

Im Folgenden gehe ich auf die Frage nach der Systematisierung oder Grammatikalisierung von ein in Otfrids Korpus ein.

\section{Systematisierung oder Grammatikalisierung von ein?}

Der Gebrauch von ein in Otfrids Korpus wird herkömmlich im Zusammenhang mit der Grammatikalisierung der Definitheit interpretiert (2.1.). Ich habe versucht zu zeigen (vgl. Pasques 2015), dass die beobachteten Fakten sich innerhalb der Nominalkategorie Numerus besser erklären lassen (2.2.). Geht es innerhalb des Numerus eher um die Systematisierung des Gebrauchs von ein als Marker des SG2, oder schon um dessen Grammatikalisierung (2.3.)?

\subsection{Die herkömmliche Interpretation von ein als Indefinitheitsmarker}

Der Sprachhistoriker Eduard Sievers behauptet, dass der Indefinitartikel ein schon im Tatian belegt ist (vgl. Sievers 1923: 316). Als Indefinitartikel betrachtet er die vier Okkurrenzen von ein ohne lateinische Entsprechung, vgl. z.B. Anmerkung 9, wo ahd. ein centenari lat. Øcenturio übersetzt. Allein der Vergleich mit dem Latein reicht aber nicht aus, um den Artikelstatus von ein zu rechtfertigen. Schon deshalb, weil sich ahd. Werke ohne lat. Quelle (wie z.B. Otfrids Evangeli-

14 In Pasques (2015) gehe ich auf diejenigen Okkurrenzen ein, die bei Otfrid trotz der Form $\emptyset N$ auf einen spezifisch zu interpretierenden SG2 hinweisen, wie z.B.:

Thar lag oba Øfélisa (Otfrid III 24 65) „Darüber lag ein Stein“; oder noch:

Er sélbo tho giméinta thar Øhórngibruader héilta (Otfrid II 24 9) „Er beschloss nun dort, einen Mann vom Aussatz zu heilen“.

In solchen Beispielen wirkt der Kontext selbstverständlich immer aktualisierend (u.a. durch Modus und Tempus). 
enharmonie) auf diese Weise nicht untersuchen lassen. Weiter, weil die Fakten innerhalb des ahd. Sprachsystems erklärt werden sollen, und nicht (nur) kontrastiv durch den Sprachvergleich. Und warum sollte überhaupt in diesem Sprachzustand ein als Marker der Indefinitheit betrachtet werden, wenn die überwältigende Mehrheit der als indefinit zu interpretierenden NG die Form $\emptyset N$ aufweist?

Erika Oubouzar schlägt einen Mittelweg vor, indem sie ein ab dem Tatian zwar nicht als Indefinitartikel betrachtet, aber als Mischform zwischen Numerale und Artikel:

Ein „drückt zwar immer noch eine Einermenge aus, dient aber der Spezifizierung. Man könnte es quantifizierendes spezifizierendes Determinativ nennen. Es hat also eine gewisse Grammatikalisierung erfahren, die aber nicht so weit geht, dass es zur Markierung indefiniter NG verwendet wird“ (Oubouzar 2000: 262).

Der quantifizierenden Funktion von ein kommt Oubouzar nach eine semantisch spezifizierende Funktion zu, die eine deiktische Komponente voraussetzt.

Mir scheint, dass auch diese Analyse von ein dessen Funktion überbewertet. Zwar liegt eine Korrelation zwischen der Verwendung von ein und der Spezifizität des entsprechenden Diskursobjektes vor. Warum wäre aber das Morphem ein als (einziger) Auslöser dieser Spezifizität zu betrachten?

Es gibt im Tatian einerseits Okkurrenzen von ein $N$, die nicht spezifisch $\mathrm{zu}$ interpretieren sind (vgl. Anm. 12, wo die NG ein man auf einen einzigen, beliebigen Mann zielt). Weiter gibt es im Tatian extrem zahlreiche, bei Otfrid zwar viel weniger Belege der Nominalsequenz $\varnothing N$, die im entsprechenden Kontext spezifisch zu interpretieren sind (vgl. Anm. 14). Im Ahd. herrscht noch das in Kapitel 1 dargestellte Prinzip: Die sog. Spezifizität bzw. Unspezifizität der Diskursobjekte ist als Auswirkung des (des-)aktualisierenden Kontextes zu betrachten; sie wird noch nicht auf der Ebene der NG enkodiert. In diesem Sinne kommt ein ab dem Tatian noch keine spezifizierende Funktion zu, wie es Oubouzar behauptet, sondern dieses Morphem ist noch als Numerale zu betrachten. ${ }^{15}$

Nicht ein allein, sondern alle an der Äußerung beteiligten Zeichen nehmen an der (Des-)Aktualisierung teil und tragen dazu bei, eine (un)spezifische Interpretation des SG2 zum Vorschein zu bringen. Das Morphem ein ist also nicht als Mischform zwischen Numerale und Indefinitartikel im Dienste der Markierung der Spezifizität zu betrachten.

15 Hier sei schon vermerkt, dass eben der semantische Wert des Numerales eine Kompatibilität mit der Aktualisierung voraussetzt, insofern als das, was man zählen kann, dazu neigt, als aktuell vorgestellt zu werden (vgl. 2.3.2). 


\subsection{Ein neuer Interpretationsversuch von ein innerhalb der Nominalkategorie Numerus}

Ist die Spezifizität als Auswirkung des Kontextes zu betrachten, so braucht man ein anderes Paradigma, um den zunehmenden Gebrauch von ein im Ahd. zu erklären. Da erweist sich die Opposition SG1 SG2 bzw. nicht diskret diskret als besonders effizient.

Im Isidortraktat und zum großen Teil noch im Tatian wird der SG2 bzw. die Diskretheit des Referenten nicht explizit markiert, sondern ist aus dem Kontext sowie dem Bibel- und Weltwissen zu rekonstruieren. Ab Otfrid aber tritt ein als explizite Markierung des SG2 immer öfter auf - in aktualisierenden Kontexten ja quasi systematisch (siehe unter 2.3.1 die quantitativen Verhältnisse). In diesem Sinne soll ein als Mischform zwischen Numerale und Marker des SG2 betrachtet werden. Beide Funktionen schließen sich nicht aus, denn der numerale Wert impliziert die Diskretheit, und die Diskretheit im Singular setzt die Einheit voraus. Aber es liegt eine Verlagerung zwischen den Werten 'Einheit' und 'Diskretheit' vor, die mit einer formellen Differenzierung einhergeht: Die akzentuierte Form éin fungiert als Numerale, die viel häufigere, nichtakzentuierte Form ein als Marker des SG2. ${ }^{16}$

Bei Otfrid ist also die Funktion von ein innerhalb der Nominalkategorie Numerus zu erklären. Kontextabhängigkeit liegt noch vor:

- In desaktualisierendem Kontext drückt ein die Einermenge aus bzw. fungiert ausschließlich als Numerale (meistens mit Akzentuierung); soll der numerale Wert der Einheit nicht in den Vordergrund treten, dann bleibt ein in solchem Kontext immer aus; ${ }^{17}$

- In aktualisierendem Kontext tritt bei Otfrid ein entweder als Numerale (mit Wert der Einheit im Vordergrund) oder als Marker der Diskretheit (SG2) auf; als Marker des SG2 wird ein eher nicht akzentuiert.

16 Akzentuierung des Numerales, Nichtakzentuierung des Markers des SG2 sind in der Handschrift nicht systematisch durchgeführt, sondern als Tendenz zu beobachten (siehe Pasques 2015).

17 Nur als Numerale ist ein in desaktualisierenden Kontexten zu beobachten; bevor ein als Marker der Diskretheit in aktualisierendem Kontext zum Zwang geworden ist, ist es mit dieser Funktion in desaktualisierendem Kontext kaum belegt (vgl. 2.3.2). 


\subsection{Systematisierung oder Grammatikalisierung von ein innerhalb der Nominalkategorie Numerus?}

Systematisierung und Grammatikalisierung sollen an dieser Stelle zunächst kontrastiv definiert werden. Unter Systematisierung verstehe ich einen sog. Obligatorisierungsprozess, ${ }^{18}$ der ohne semantisch-funktionellen Wandel erfolgt (oder erfolgen kann). Die Markierung einer kategoriellen Opposition, die früher nicht explizit und/oder nicht systematisch erfolgte, wird somit zum Zwang, ohne dass den Oppositionsmarkern eine grundsätzlich neue Funktion zukommt (oder zukommen muss). Grammatikalisierung hingegen setzt sowohl Obligatorisierung als auch Desemantisierung (oder eine andere semantisch-funktionelle Veränderung) ${ }^{19}$ der Oppositionsmarker voraus.

Um den Unterschied zwischen beiden Prozessen klarzumachen, möchte ich den Grammatikalisierungsprozess vom Morphem ther ('der') sehr kurz erwähnen, wie ihn Szczepaniak/Flick (2015) beschreiben. ${ }^{20}$ Im Isidortraktat ist ther mal als Demonstrativ, mal schon als Marker der Definitheit aufzuwerten. Zwischen beiden Funktionen liegt ein wesentlicher semantisch-funktioneller Wandel vor, wie ihn die entsprechende Korpusuntersuchung aufdeckt, der auf die einsetzende, sich erst im Anfangsstadium befindende Grammatikalisierung von ther zurückzuführen ist.

18 Den Terminus „Obligatorisierung“ verwende ich in Anlehnung an Szczepaniak/Flick (2015: 190).

19 Die entsprechende semantisch-funktionelle Veränderung, die einen Grammatikalisierungsprozess kennzeichnet, wird herkömmlich als Übergang aus der lexikalischen in die grammatische Ebene oder aus einer weniger grammatischen in eine mehr grammatische Ebene verstanden, vgl. Kurylowicz (1965: 69): „Grammaticalization consists in the increase of the range of a morphem advancing from a lexical to a grammatical or from a less grammatical to a more grammatical status“. Für eine Synthese der verschiedenen Grammatikalisierungstheorien vgl. Szczepaniak (2011).

20 „Im Isidor befindet sich die Grammatikalisierung des Demonstrativs zum Definitartikel im Anfangsstadium. Die Definitheit muss also in den meisten Fällen kovert markiert werden. Im Kontext der adnominalen Genitive zeichnet sich jedoch eine Desemantisierung von ther ab. Warum dieser Wandel in diesem Kontext so früh eintritt, kann möglicherweise mit der besonderen Rolle der adnominalen (nicht-partitiven) Genitive erklärt werden [...]. Vermutlich ist die frühe Entwicklung des Definitartikels damit zu erklären, dass attributive Genitive ein häufiges Mittel zum verdeckten Ausdruck der Definitheit sind. In diesen Kontexten kommt es also verstärkt zur inflationären Verwendung von ther, der zunächst zur Verdeutlichung der definiten Lesart dienen soll. Dies bewirkt anschließend seine Desemantisierung“ (Szczepaniak/Flick 2015: 203; Unterstreichung von mir). 
Ich möchte anhand von quantitativen und qualitativen Fakten im Folgenden fragen, ob im Falle von ein als Marker der Diskretheit (bzw. innerhalb der Kategorie des Numerus, und nicht der Definitheit) Obligatorisierung mit oder ohne Desemantisierung vorliegt.

\subsubsection{Die quantitativen Verhältnisse}

Die quantitativen Verhältnisse im Korpus Otfrids sind folgende. ${ }^{21}$ Das Morphem ein taucht 46-mal in einer singularischen, ${ }^{22}$ nicht pronominalen, ${ }^{23}$ nicht definiten $\mathrm{NG}^{24}$ auf. Die Nominalsequenz $\emptyset N$ dagegen ist für den spezifischen SG2 (d.h. in einem aktualisierenden Kontext) 4-mal, für den unspezifischen SG2 (d.h. in einem desaktualisierenden Kontext) 22-mal zu verzeichnen. ${ }^{25}$

Das Stadium der Systematisierung des Gebrauchs von ein als Marker des SG2 ist also noch nicht erreicht, mit anderen Worten der Obligatorisierungsprozess ist noch nicht vollzogen, denn vier Belege der Nominalsequenz $\emptyset N$ entsprechen noch einem spezifischen SG2. Wirkt der Kontext aktualisierend, bzw. geht es um ein zeitlich-räumlich verankertes, diskretes Diskursobjekt, so tritt ein in Otfrids Korpus noch nicht systematisch auf. In desaktualisierendem Kontext ist ein kaum belegt. Kontextabhängigkeit herrscht auf jeden Fall noch vor.

Im Vergleich zum Tatian, wo der Gebrauch von ein auch in aktualisierendem Kontext die Ausnahme ist, und bis auf vier Belege lat. unus/quidam/solus übersetzt, haben sich aber die quantitativen Verhältnisse in Otfrids Korpus so gewandelt, dass der Obligatorisierungsprozess von ein als Marker des aktuellen SG2 als sehr fortgeschritten zu betrachten ist. Die grammatische Innovation ab Otfrid

21 Das untersuchte Korpus besteht aus dem dritten Buch der Evangelienharmonie Otfrids. 22 In diesem Korpus liegt ein auch in NG im Plural vor (vgl. frz. quelques-uns), wie z.B. in: Sih náhtun eino zíti [PL] thaz man tho fíroti eina wéchun thuruh nót ... (Otfrid III 15 5) „Da nahte[n] sich nun jene Zeit[en], dass man eine Woche lang feierte ...“

23 Das Morphem ein taucht auch als Pronomen auf:

Thaz báz ist man biwérbe, thaz éin man bi unsih stérbe, joh éiner bi unsih dówe ... (Otfrid III 25 25-26) ,... dass besser ist, dass ein [einziger] Mann für uns stirbt, dass einer für uns verdirbt ...“

24 Das Morphem ein taucht auch in definiten NG auf, meistens mit numeralem Wert, wie in: Er ríat thaz man biwúrbi, thaz ther man éino irstúrbi, thaz sin éinen dóti al then líut gihíalti (Otfrid III 25 27) „Er riet, dass man es ausführte, dass dieser eine Mensch sterbe, damit des einen Untergang das ganze Volk errette“.

25 Nicht mitberücksichtigt sind die singularischen, nicht definiten NG, die zwar dem SG2 entsprechen, aber weiter determiniert werden (Ømihil ungiwitiri „ein großes Gewitter“; Øbrunno thes bluates „die Quelle des Blutes“). 
besteht also quantitativ in der zunehmenden Obligatorisierung der Markierung für die bisher nicht explizit markierte, aber sehr wahrscheinlich schon existierende Diskretheit im Rahmen der kategoriellen Opposition nicht diskret $\sim$ diskret. ${ }^{26}$

\subsubsection{Die qualitativen Verhältnisse}

Qualitativ bzw. semantisch soll gefragt werden, ob zwischen ein als Numerale und ein als Marker der Diskretheit eine semantisch-funktionelle Verlagerung zu beobachten ist, die auf einen Grammatikalisierungsprozess schließen lässt oder nicht.

Die alte, herkömmliche Funktion von ein ist die quantifizierende. Die Verwendungen von ein als Numerale, fast nur in aktualisierenden Kontexten belegt, sind über die Akzentuierung hinaus dadurch zu erkennen, dass in den entsprechenden Textstellen sehr oft eine Einheit einer Mehrheit gegenübergestellt wird. Die sich ab Otfrid abzeichnende Funktion von ein als Marker der Diskretheit ersetzt die herkömmliche quantifizierende nicht, sondern kommt sozusagen hinzu. Ich meine die zahlreichen Textstellen, wo der numerale Wert von ein nicht ganz relevant ist und ein nicht mehr die Einheit als Gegenteil von Zweiheit oder Mehrheit zum Ausdruck bringt, sondern die Diskretheit als Gegenteil vom Kontinuierlichen signalisiert. Es liegt auf jeden Fall im untersuchten Korpus zwischen 'Einheit' und 'Diskretheit' ein semantisches Kontinuum vor, das Schwierigkeiten auslösen kann, versucht man zwischen beiden Werten zu unterscheiden.

Über dieses semantische Kontinuum hinaus ist aber die schon erwähnte Kontextabhängigkeit des Gebrauches von ein für die vorliegende Fragestellung sehr aufschlussreich. Dass ein bei Otfrid kaum in desaktualisierenden Kontexten verwendet wird, lässt sich nämlich semantisch erklären: Die Existenzpräsupposition, ${ }^{27}$ die jedem Numerale anhaftet, ist mit Desaktualisierung nicht kompatibel. ${ }^{28}$ Mit anderen Worten, ein ist als Numerale noch nicht desemantisiert, es

26 Die Nicht-Markierung der kategoriellen Opposition nicht diskret diskret (oder SG1 SG2) vor Otfrid kann keinesfalls mit der Nicht-Existenz dieser Opposition gleichgesetzt werden. Diese Opposition wird in früheren Texten durch Kontext und Weltwissen (ja Bibelwissen) inferiert, oder auch durch andere Zeichen markiert (vgl. Pasques 2015).

27 Der Zusammenhang zwischen Einheit, Diskretheit, Form einerseits und Existenz andererseits besteht darin, dass das, was man zählen kann, auch räumlich verankert ist bzw. zur Existenz neigt. Bergson (2013 [1927]: 66) spricht in diesem Sinne von solidarité des notions de nombre et d'espace („Solidarität der Begriffe von Zahl und Raum“), vgl. Pasques (2015: 55).

28 Stark beobachtet dieselben Verhältnisse im Altitalienischen: „Spezifisch interpretierbare NG [neigen] eher zur Determinierung als nicht-spezifische [...]. Das Auftreten des Numerales 'eins' 
taucht deshalb nur in aktualisierendem Kontext auf. In diesem Sinne schlage ich vor, für dieses Korpus von einem einsetzenden Obligatorisierungsprozess zu sprechen, der noch ohne Desemantisierung von ein einhergeht bzw. nicht als Grammatikalisierung aufzufassen ist, da die Systematisierung der Verwendung von ein für den SG2 nur in aktualisierenden Kontexten zu beobachten ist.

Diese Existenzpräsupposition von ein als Marker der Einheit und der Diskretheit erweist sich als besonders gut geeignet, in narrativen Textstellen ${ }^{29}$ neue Diskursobjekte einzuführen (vgl. Pasques 2015). Der Obligatorisierungsprozess ist deshalb dann am weitesten fortgeschritten, wenn die singularische, nicht definite NG als erstes Glied einer Referenzkette vorkommt. In solchem Kontext ist über den numeralen Wert von ein hinaus dessen noch intakt erhaltene Existenzpräsupposition relevant, die es erlaubt, Form und Existenz des neueingeführten Referenten $\mathrm{zu}$ indizieren.

\section{Schluss}

Ab Otfrid lässt sich hinsichtlich der Funktion von ein und der Frage nach dessen Systematisierung bzw. Obligatorisierung oder Grammatikalisierung Folgendes behaupten:

- Innerhalb der Nominalkategorie Numerus wird ein quasi systematisch verwendet, um den SG2 in aktualisierendem Kontext zu markieren. Das Diskursobjekt, auf das durch ein $N$ verwiesen wird, wird als diskrete, im entsprechendem Kontext meistens als spezifisch $\mathrm{zu}$ interpretierende Einheit hingestellt.

- Berücksichtigt man die Korrelation zwischen Verwendung von ein und Aktualisierung des entsprechenden SG2, so liegt es nahe anzunehmen, dass ein als aktualisierendes Zeichen, ja als einziger Auslöser der Aktualisierung des SG2 zu betrachten ist. Die im Korpus beobachteten Fakten lassen diese Annahme aber nicht zu. Die Wechselbeziehungen zwischen Verwendung von ein und Semantik des Kontextes dürfen in diesem Sprachzustand keineswegs übersehen werden.

[kann] umgekehrt in nicht-spezifischen Kontexten als Indikator für seinen Artikelstatus gewertet werden“ (Stark 2006: 118, Anm. 175).

29 Diese Funktion ist in narrativen Textstellen zu finden, die im Isidortraktat und im Tatian viel seltener sind. 
- Das semantische Kontinuum zwischen Einheit und Diskretheit einerseits, das Nicht-Auftreten von ein in desaktualisierenden Kontexten andererseits, das auf die Existenzpräsupposition des Numerales bzw. auf die NichtDesemantisierung von ein zurückzuführen ist, erlauben es nicht, über den einsetzenden Obligatorisierungsprozess hinaus auch einen Grammatikalisierungsprozess anzunehmen, der einen semantisch-funktionellen Wandel von ein voraussetzt.

- Die neue textuelle Funktion von ein, die sich in den narrativen Textstellen von Otfrids Evangelienharmonie sehr regelmäßig beobachten lässt, ergibt sich aus der Existenzpräsupposition des Numerales ein: In solchen Kontexten markiert ein die Ersteinführung eines Diskursobjektes, und dher dessen Wiederaufnahme. Wahrscheinlich erst im Mittelhochdeutschen löst eben diese Textfunktion von ein dessen Reinterpretation als Pendant zu der innerhalb der Nominalkategorie der Definitheit aus (vgl. Desportes 2000).

\section{Literatur}

Bergson, Henri (2013 [1927]): Essai sur les données immédiates de la conscience. Paris: Presses Universitaires de France (PUF).

Briu, Jean-Jacques (2001): Les groupes syntaxiques en allemand par J. Fourquet (Sorbonne, 1966, polycopié restitué). In: Histoire Épistémologie Langage 23, 1. 153-182. (www.persee. $\mathrm{fr} / \mathrm{doc} / \mathrm{hel}$, Stand: 23.11.2017).

Desportes, Yvon (2000): Der Artikel im Mittelhochdeutschen. Lässt sich Paul Valentins Modell des Artikelsystems im heutigen Deutsch auf das Mittelhochdeutsche übertragen? In: Desportes, Yvon (Hg.): Zur Geschichte der Nominalgruppe im älteren Deutsch. Festschrift für Paul Valentin. Heidelberg: Winter. 213-253.

Eggers, Hans (Hg.) (1964): Der althochdeutsche Isidor nach der Pariser Handschrift und den Monseer Fragmenten. Tübingen: Niemeyer.

Erdmann, Oskar (Hg.) (1882/1973): Otfrids Evangelienbuch, 6. Aufl. besorgt v. Ludwig Wolff, Altdeutsche Textbibliothek 49. Tübingen: Niemeyer.

Fourquet, Jean (1992): Sémantique catégorielle et sémantique connexionnelle. In: Gréciano, Gertrud/Kleiber, Georges (Hgg.): Systèmes Interactifs. Mélanges en l'honneur de Jean David. Paris: Klincksieck. 141-156.

Kleiber, Georges (1989): Le Générique, un massif? In: Langages 94, 24. 73-113.

Kurylowicz, Jerzy (1965): The evolution of grammatical categories. In: Diogenes 51, 13. 55-71.

Martinet, André (1970): Eléments de linguistique générale. Paris: Armand Colin.

Masser, Achim (Hg.) (1994): Die lateinisch-althochdeutsche Tatianbilingue. Stiftsbibliothek St. Gallen Cod. 56. (= Studien zum Althochdeutschen 25). Göttingen: Vandenhoeck \& Ruprecht.

Oubouzar, Erika (2000): Zur Entwicklung von ein in der Nominalgruppe des Althochdeutschen. In: Desportes, Yvon (Hg.): Zur Geschichte der Nominalgruppe im älteren Deutsch. Festschrift für Paul Valentin. Heidelberg: Winter. 255-268. 
Pasques, Delphine (2015): Contribution à l'histoire de la catégorie du Numerus en ancien-hautallemand: L'émergence du marquage nominal de l'opposition discret non discret. Paris: Université Paris Sorbonne/Montpellier III. [Habilitationsschrift].

Presslich, Marion (2000): Partitivität und Indefinitheit. Die Entstehung und Entwicklung des indefiniten Artikels in den germanischen und romanischen Sprachen am Beispiel des Deutschen, Niederländischen, Französischen und Italienischen. Frankfurt a.M.: Lang.

Sievers, Eduard (1923): Tatian. Lateinisch und Altdeutsch mit ausführlichem Glossar. Paderborn: Schöningh.

Soutet, Olivier (1995): Linguistique. Paris: PUF.

Szczepaniak, Renata (2011): Grammatikalisierung im Deutschen. Tübingen: Narr.

Sczcepaniak, Renata/Flick, Johanna (2015): Zwischen Explizitheit und Ökonomie - Der emergierende Definitartikel in der althochdeutschen Isidor-Übersetzung. In: Pasques, Delphine (Hg.): Komplexität und Emergenz in der deutschen Syntax (9.-17. Jahrhundert). Berlin: Weidler. 187-206.

Stark, Elisabeth (2006): Indefinitheit und Textkohärenz. Entstehung und Textstrukturierung indefiniter Nominaldetermination im Altitalienischen. Tübingen: Niemeyer.

Thormann, Kurt (2013): Destillieren und Rektifizieren. Berlin: Springer-Verlag.

Valentin, Paul (1984): Le jeu du nombre et de la définitude dans le GN allemand. In: David, Jean/Kleiber, Georges (Hgg.): Déterminants: syntaxe et sémantique. Metz: Centre d'analyse linguistique de l'Université de Metz. 263-281. 